\title{
Defective Neutrophil Oxidative Burst in Preterm Newborns on Exposure to Coagulase-Negative Staphylococci
}

\author{
MARIA BJÖRKQVIST, MARGARETHA JURSTRAND, LENNART BODIN, HANS FREDLUND, AND \\ JENS SCHOLLIN \\ Departments of Pediatrics [M.B., J.S.] and Clinical Microbiology [M.J., H.F.] and Clinical Research \\ Center [L.B.], Örebro University Hospital, S-701 85 Örebro, Sweden
}

\begin{abstract}
The neutrophil oxidative burst is a product of the regulated assembly of the multicomponent oxidase enzyme. Our aim was to compare the oxidative burst in term $(n=10)$ and preterm newborns $<31$ wk gestational age $(n=10)$ after stimulation with coagulase-negative staphylococci in vitro. Strains of Streptococcus epidermidis with different invasive and slime-producing properties, one strain of $S$. haemolyticus, and one strain of group B-streptococcus were investigated. A whole-blood flow cytometric assay using the oxidation of hydroethidine to ethidium bromide was used. The oxidative activity in unstimulated neutrophil granulocytes [polymorphonuclear leukocytes (PMNLs)] was similar in term and preterm newborns, but the preterm newborns showed a significantly lower capacity to up-regulate the oxidative burst intensity after bacterial stimulation $(p=0.004)$. In the term but not in the preterm group, the oxidative burst intensity after bacterial stimulation correlated with the baseline oxidative burst intensity. After bacterial stimulation, there was a trend
\end{abstract}

toward a greater percentage of activated neutrophils in the term group than in the preterm group, but the difference was less pronounced than that in oxidative burst intensity. Significant differences in oxidative burst response to different bacterial strains were observed $(p<0.001)$, but the differences could not be correlated exclusively to invasive capacity or slime-producing properties. It is concluded that the baseline oxidative activity is similar in term and preterm PMNLs but that preterm PMNLs have a decreased capacity to increase the oxidative burst in response to bacterial stimulation. (Pediatr Res 55: 966-971, 2004)
Abbreviations
CoNS, coagulase-negative staphylococci
GBS, group B streptococcus
PMNL, polymorphonuclear leukocyte

Decreased activity in both humoral and cellular defense mechanisms contributes to the increased risk of invasive infections in newborn infants. Impaired neutrophil production and function in newborn infants are well documented (1); however, reports concerning neonatal phagocytosis and subsequent intracellular oxidative bacterial killing have been conflicting (2-4).

Coagulase-negative staphylococci (CoNS) are the major cause of neonatal septicemia in very low birth weight newborns $(<1500 \mathrm{~g})(5)$. The production of extracellular slime (biofilm) has been proposed as a major virulence factor of Streptococcus epidermidis, although this issue is still under debate $(2,6)$. In a previous study of neonatal blood isolates of CoNS, we found that one genotype predominated among the

Received January 14, 2003; accepted January 6, 2004.

Correspondence: Maria Björkqvist, Ph.D., Department of Pediatrics, Örebro University Hospital, S-701 85 Örebro, Sweden; e-mail: maria.bjorkqvist@orebroll.se

This study was supported by grants from the Research Committee of Örebro County Council, Sweden.

DOI: 10.1203/01.pdr.0000127018.44938.89 sepsis strains of $S$. epidermidis but not among the strains classified as contaminants. S. haemolyticus selectively colonized and infected the most preterm infants (7).

The neutrophil bacterial killing is mediated by extracellular release of bactericidal proteins from the neutrophil secondary granulae and by intracellular oxidative and nonoxidative mechanisms (8). The neutrophil oxidative burst is a product of the regulated assembly of the multicomponent oxidase enzyme (9). Flow cytometric methods permit investigation of the oxidative burst in a whole-blood system, thus avoiding the procedure of preparing granulocyte samples and allowing investigation of very small blood sample volumes $(10,11)$. Quantification of the oxidative burst using the oxidation of hydroethidine to ethidium bromide has been found to be closely correlated to the number of phagocytosed bacteria and thus can be used as an indirect measurement of phagocytosis (12). Hydroethidine is readily transferred into the cell and is then oxidized to ethidium bromide at an early stage in the oxidative burst chain. Because ethidium bromide does not cross the cell membrane, only intracellular reactions are assessed (13). 
In most previous studies of the oxidative burst in newborns, formylmethionyl-leucyl-phenylalanine, phorbol myristate acetate, or other synthetic agents have been used for neutrophil stimulation (14-17). The aim of the present study was to investigate the neutrophil oxidative burst after stimulation with CoNS in term and very low birth weight newborns and to compare the oxidative response after stimulation with invasive and noninvasive bacterial strains.

\section{METHODS}

Patients. Premature but otherwise healthy newborns who had a gestational age of $<31 \mathrm{wk}$ and were admitted to the neonatal intensive care unit of University Hospital (Örebro, Sweden) between April 2001 and January 2002 were recruited at 2-7 d of age. Exclusion criteria were signs of infection in the child or the mother, treatment with i.v. gammaglobulin or fresh frozen plasma, or respiratory distress requiring mechanical ventilation on the day of the study.

For the oxidative burst assay, $1 \mathrm{~mL}$ of blood was collected through an indwelling arterial umbilical catheter and distributed into a Na-Heparin tube (367671; Becton Dickinson, Franklin Lakes, NJ, U.S.A.; 30 USP heparin/mL). The sample was immediately transferred at room temperature to the laboratory for further investigation. Arterial blood, $0.25 \mathrm{~mL}$, collected into a Microtainer EDTA tube (365955; Becton Dickinson) was obtained at the same time for determination of the total neutrophil count.

One healthy, term newborn who had a gestational age of $38-41 \mathrm{wk}$ and Apgar $>7$ at $5 \mathrm{~min}$ and was delivered after an uncomplicated pregnancy was recruited on each experimental occasion together with the premature newborn. Blood was collected simultaneously with the routine screening sampling for metabolic diseases at 3-5 d of age. The same volumes and tubes as for the premature newborns were used.

Control. One and the same healthy adult was included as a control in every experiment. The same blood sample volumes and test tubes as for the newborns were used.

Bacteria. From a former study of neonatal blood, isolates of CoNS strains with different genotypes and biochemical profiles were selected (7). After determination of slime production (see below), four strains of $S$. epidermidis were selected for the oxidative burst assay, together with one invasive strain of $S$. haemolyticus and one invasive strain of group B streptococcus (GBS) (Table 1). The strains of $S$. epidermidis were selected to allow collection of isolates representing both sepsis and contaminant strains, slimeproducing and slime-negative strains, and strains with dif- ferent genotypes and biochemical profiles. One of the sepsis isolates of S. epidermidis (94B080) represented the dominating genotype of the invasive CoNS strains in the former study (genotype A), whereas the other sepsis isolate (94B105) represented a unique genotype (single isolate). The isolate of $S$. haemolyticus represented an endemic strain identified on our neonatal ward in the former study. The GBS strain, capsular serotype III, represented a highly virulent clinical isolate from a newborn with sepsis and meningitis (18). All bacteria were stored at $-70^{\circ} \mathrm{C}$ pending further investigation.

Determination of slime production. Slime production by the strains of $S$. epidermidis was determined after $24 \mathrm{~h}$ of culture in tryptic soy broth with $0.25 \%$ glucose and staining with Trypan blue according to the method described by Mulder et al. (19). Three reference strains of $S$. epidermidis were used as controls in the slime determination assay: ATCC 35982 (slimenegative), ATCC 35983 (slime ++ ), and ATCC 35984 (slime $+++)$.

Bacteria for oxidative burst assay. For the oxidative burst assay, bacteria were cultivated on blood agar medium $(4.25 \%$ Colombia II Agar; BBL Becton Dickinson and Company, Baltimore, MD, U.S.A.; 0.3\% Agar No. 2 LabM, UK; 5\% bovine blood) for $24 \mathrm{~h}$ at $37^{\circ} \mathrm{C}$, recultivated, incubated for another $24 \mathrm{~h}$ at $37^{\circ} \mathrm{C}$, and checked for purity. The GBS strain was incubated in $5 \% \mathrm{CO}_{2}$ at $37^{\circ} \mathrm{C}$. Each bacterial strain was then suspended in PBS, washed once, and resuspended in PBS. After counting in a Bürker chamber, the bacterial density was adjusted to 10 bacteria per polymorphonuclear leukocyte (PMNL) for each study person.

Oxidative burst assay and flow cytometry. The neutrophil oxidative burst response was assessed with use of hydroethidine (Molecular Probes, Leyden, Netherlands) according to the method described by Kragsbjerg et al. (20) with minor modifications. Hydroethidine was dissolved in DMSO at a concentration of $1 \mathrm{mg} / \mathrm{mL}$ and stored at $-70^{\circ} \mathrm{C}$. Fifty microliters of heparinized whole blood was mixed with $10 \mu \mathrm{L}$ of hydroethidine $0.01 \mathrm{mg} / \mathrm{mL}$ in PBS in plastic tubes (Falcon 2058; Becton Dickinson) and incubated at $37^{\circ} \mathrm{C}$ for $15 \mathrm{~min}$ under mild agitation. Two sets of tubes for each bacterial strain and for each participating individual were prepared. Ten microliters of bacterial suspension was added. Two tubes with whole blood and hydroethidine for each study person served as controls. The tubes were incubated at $37^{\circ} \mathrm{C}$ under mild agitation for 0 and $30 \mathrm{~min}$, and the incubations were then terminated by placing the tubes on ice. The samples were lysed and fixed using $1 \mathrm{~mL}$ of FACS Lysing Solution 10\% (Becton Dickinson)

Table 1. Clinical and bacteriologic data of the bacterial strains used in the oxidative burst assay

\begin{tabular}{|c|c|c|c|c|}
\hline Identity & Species & Clinical data & Genotype & Slime \\
\hline $94 \mathrm{~B} 105$ & S. epidermidis & Sepsis & Single isolate & +++ \\
\hline $94 \mathrm{~B} 080$ & S. epidermidis & Sepsis & A & Negative \\
\hline $95 \mathrm{~B} 523$ & S. epidermidis & Contaminant & E & Negative \\
\hline $91 B 735$ & S. epidermidis & Contaminant & Single isolate & ++ \\
\hline $95 \mathrm{~B} 535$ & S. haemolyticus & Sepsis & $\mathrm{C}$ & Negative \\
\hline GBS VII & GBS & Sepsis and meningitis & & \\
\hline
\end{tabular}

The characterization of the strains, including genotyping of the isolates of CoNS, refers to previous studies 7,18 . 


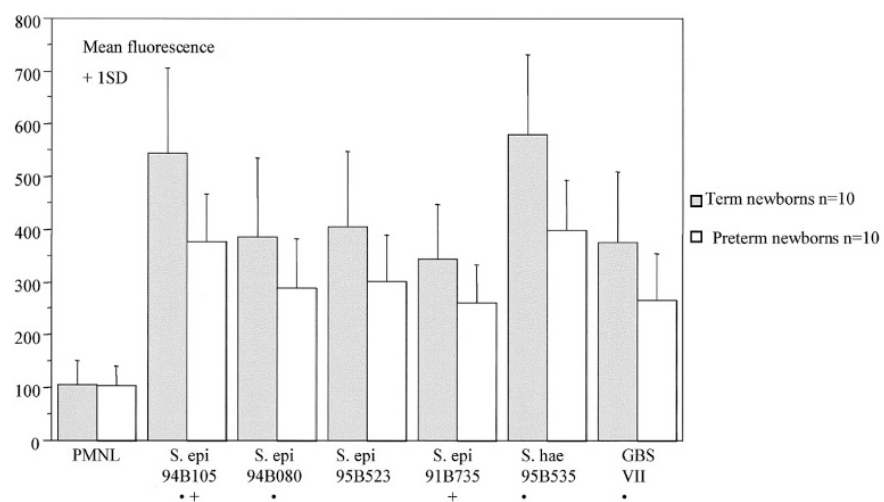

Figure 1. Oxidative burst intensity, expressed as mean fluorescence, in neonatal PMNLs after $30 \mathrm{~min}$ of incubation with different neonatal blood isolates of bacteria. ', sepsis strains; +, production of slime; S. epi, $S$. epidermidis; S. hae, S. haemolyticus.

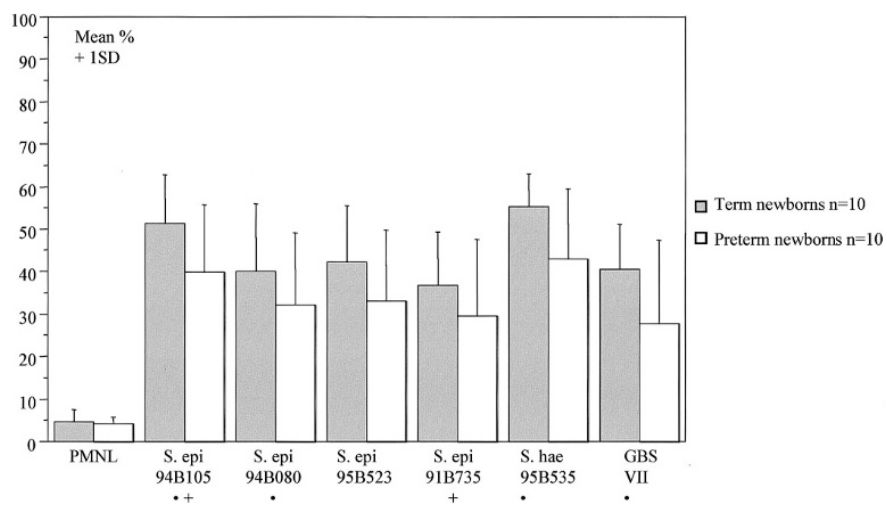

Figure 2. Percentage of neonatal neutrophils showing oxidative activity after 30 min of incubation with different neonatal blood isolates of bacteria. ', sepsis strains; +, production of slime; S. epi, S. epidermidis; S. hae, S. haemolyticus; GBS, Group B streptococcus.

at room temperature for $10 \mathrm{~min}$. After centrifugation $(300 \times g$, $5 \mathrm{~min}$ ) and removal of the supernatant, the pellet was resuspended in $1.5 \mathrm{~mL}$ of PBS with $1 \%$ formaldehyde, centrifuged, and resuspended in $0.4 \mathrm{~mL}$ PBS with $1 \%$ formaldehyde. The tubes were then stored at $4^{\circ} \mathrm{C}$ for a maximum of $18 \mathrm{~h}$ until analyzed.

Flow cytometry analyses were performed using a FACScan flow cytometer (Becton Dickinson Immunocytometric Systems, San José, CA, U.S.A.) with computer-assisted evaluation of data (Cellquest software; Becton Dickinson). The instrument was set by means of the AutoComp test with minor modifications: the forward scatter threshold was raised to 180 , and the sideward scatter threshold was lowered to 370 . The fluorescence parameters from single cells were collected after gating using a combination of forward and sideward scatter. A total of 5000 PMNLs were analyzed per tube. The intensity of the oxidative burst was assessed on the basis of the mean fluorescence. The percentage of activated neutrophils was determined as events of fluorescence $\times 100 / 5000$ PMNLs.

Statistical methods. Statistical methods based on within-pair comparisons were used, and the observational unit in focus was the pair of newborns, one preterm and one term. Differences in mean fluorescence and in percentage of activated neutrophils by gestational age (group) or bacterial strain were assessed, using an ANOVA model for repeated measurements with two within-pair factors, i.e. group and strain, and an interaction factor (group*strain). In a first step, all strains were analyzed simultaneously, with use of a multivariate ANOVA (MANOVA) model. A univariate analysis with paired $t$ test of the differences in mean fluorescence and percentage of activated neutrophils was used for further comparisons between the two groups of newborns. For post hoc assessments of the differences among the six bacterial strains within the two groups of newborns, pairwise comparisons with Bonferroni correction for multiple testing was used. The linear association between unstimulated PMNLs and PMNLs after bacterial stimulation, for each group of newborns separately, was estimated with Pearson's correlation coefficient. The significance level was set at $p<0.05$, and 95\% confidence intervals (CI) were constructed.

Ethics. The local ethics committee approved the study. Informed consent of the parents was obtained before recruitment of all newborns.

\section{RESULTS}

Patients. Thirteen pairs of term and preterm newborn infants were included between April 2001 and January 2002. Three preterm children developed blood culture-proven sepsis within $24 \mathrm{~h}$ after entering the study and were excluded from further evaluation, as were their corresponding term newborns. The median gestational age of the preterm newborns $(n=10)$ was $28 \mathrm{wk} \pm 4 \mathrm{~d}$ (range $24 \mathrm{w} \pm 0 \mathrm{~d}$ to $30 \mathrm{w} \pm 2 \mathrm{~d}$ ) and the median birth weight was $1038 \mathrm{~g}$ (range 735-1510 g). In the term newborns $(n=10)$ the median gestational age was $40 \mathrm{w} \pm 0 \mathrm{~d}$ (range $38 \mathrm{w} \pm 4 \mathrm{~d}$ to $41 \mathrm{w} \pm 0 \mathrm{~d}$ ) and the median birth weight was $3655 \mathrm{~g}$ (range 2660-4205 g). The age at inclusion (median $3 \mathrm{~d}$ ) was similar in the two groups: range $2-5 \mathrm{~d}$ in the preterm group and range 3-4 $\mathrm{d}$ in the term newborns.

Oxidative burst in unstimulated PMNLs in term and preterm newborns. In unstimulated PMNLs, there were no differences between the term and preterm newborns in the intensity of the oxidative burst or in the percentage of activated neutrophils. After $30 \mathrm{~min}$ of incubation without bacteria, there were still no differences between the two groups of newborns (Figs. 1 and 2, Table 2). At the start of incubation ( $0 \mathrm{~min})$, there were no differences in oxidative burst intensity or in the percentage of activated neutrophils in the tubes with the different bacterial strains compared with PMNLs without bacteria (data not shown).

Oxidative burst intensity after bacterial stimulation in term and preterm newborns. The term newborns showed a higher capacity to up-regulate the oxidative burst intensity after bacterial stimulation compared with the preterm infants. After 30 min of incubation, the mean fluorescence was significantly higher in the term newborns than in the preterm newborns for all bacterial strains (MANOVA, the group factor, $p=0.004$; Fig. 1). In univariate analyses ( $t$ test), this difference was statistically significant for all bacterial strains (Table 2).

Neutrophil activation in term and preterm newborns after bacterial stimulation. In both groups of newborns, there was a 
Table 2. Differences in oxidative burst intensity and in the percentage of activated neutrophils between term and preterm newborns after 30 min of incubation with different bacterial strains

\begin{tabular}{|c|c|c|c|c|}
\hline Strain & $\begin{array}{c}\text { Fluorescence } \\
\text { [Mean difference }(95 \% \mathrm{CI})]\end{array}$ & $p$ & $\begin{array}{c}\% \text { of activated neutrophils } \\
{[\text { Mean difference }(\%)(95 \% \mathrm{CI})]}\end{array}$ & $p$ \\
\hline PMNIs without bacteria & $2(-35-40)$ & 0.89 & $0.6(-1.6-2.8)$ & 0.56 \\
\hline S. epi*† 94B105 & $166(78-243)$ & 0.002 & $11.4(0.6-22.2)$ & 0.040 \\
\hline S. epi 95B523 & $103(23-182)$ & 0.017 & $9.2(-3.7-22.1)$ & 0.14 \\
\hline S. epi† 91B735 & $82(11-153)$ & 0.028 & $7.2(-5.4-19.8)$ & 0.23 \\
\hline S. hae* 95B535 & $181(84-278)$ & 0.002 & $12.3(1.0-23.7)$ & 0.037 \\
\hline
\end{tabular}

S. epi, S. epidermidis; S. hae, S. haemolyticus; GBS, Group B streptococcus; CI, confidence interval.

* Sepsis isolate.

$\dagger$ Slime-positive.

significant increase in the percentage of activated neutrophils after $30 \mathrm{~min}$ of incubation with every different bacterial strain (Fig. 2). The term newborns tended to show a higher percentage of activated neutrophils than the preterm group (Fig. 2), but this difference was not significant (MANOVA, the group factor, $p=0.093)$. In univariate analyses $(t$ test), significance was attained in two of the six strains (Table 2).

Oxidative burst response after stimulation with different bacterial strains. Within the two groups of newborns, significant differences between the oxidative burst responses to different bacterial strains were observed after $30 \mathrm{~min}$ of incubation. This applied both to the oxidative burst intensity (MANOVA, the strain factor, $p<0.001$ ) and to the percentage of activated neutrophils (MANOVA, the strain factor, $p<$ 0.001). However, the impact of bacterial strain seemed to differ between the two groups of newborns regarding the oxidative burst intensity, because a statistical interaction was found between group and strain (MANOVA, strain*group factor, $p=$ 0.036 ), but this was not seen for the percentage of activated neutrophils (MANOVA, strain*group factor, $p=0.362$ ). These differences could not be related exclusively to invasive properties or to slime production capacity. The slime-positive sepsis strain of $S$. epidermidis (94B105) and the strain of $S$. haemolyticus (95B535) formed a "high-response group" that displayed a comparably high response both in the oxidative burst intensity (Fig. 1) and in the percentage of activated neutrophils (Fig. 2). The other bacteria formed a "low-response group" (the slime-negative sepsis strain of $S$. epidermidis, the two contaminant strains of S. epidermidis irrespective of slime production, and the GBS-strain; Figs. 1 and 2). Pairwise comparison strain by strain showed that these differences between the strains in the high-response and low-response groups were statistically significant both within the term group and within the group of preterm neonates. There was no difference in oxidative burst intensity or in the percentage of activated neutrophils between the strains either within the high-response or within the low-response group.

Correlation of oxidative burst activity between unstimulated and stimulated neutrophils. In the term newborns, the oxidative burst intensity after stimulation with the different bacterial strains correlated to that in unstimulated PMNLs after $30 \mathrm{~min}$ of incubation. In the preterm newborns, no such correlation was found (Table 3). Regarding the percentage of activated neutrophils, the correlation between unstimulated and stimulated PMNLs in the term newborns varied according to bacterial strain but with low values. In the preterm newborns, there was a strong negative correlation between the percentage of activated neutrophils after bacterial stimulation and that in unstimulated PMNLs after 30 min of incubation (Table 3).

Control. The mean fluorescence in unstimulated PMNLs in the adult control after $30 \mathrm{~min}$ of incubation was 48.7 (SD 18.02), and the mean percentage of activated neutrophils was $3.2 \%$ (SD 1.75\%).

\section{DISCUSSION}

In the present study, preterm neonates showed a lower capacity to up-regulate the neutrophil oxidative burst after bacterial stimulation compared to term neonates. This difference in oxidative burst intensity between the two groups was highly statistically significant for all bacterial strains. The neutrophil respiratory burst represents the oxygen-dependent chain of intracellular reactions producing a number of bactericidal metabolites such as superoxide anion, hydrogen perox-

Table 3. Correlations (Pearson's) between PMNLs before and after stimulation with different bacterial strains for oxidative burst intensity and percentage of activated neutrophils

\begin{tabular}{|c|c|c|c|c|c|c|}
\hline & $\begin{array}{c}\text { S. epi } \\
\text { 94B105 }\end{array}$ & $\begin{array}{c}\text { S. epi } \\
94 \mathrm{~B} 080\end{array}$ & $\begin{array}{c}\text { S. epi } \\
\text { 91B523 }\end{array}$ & $\begin{array}{c}\text { S. epi } \\
\text { 91B735 }\end{array}$ & $\begin{array}{c}\text { S. hae } \\
\text { 95B535 }\end{array}$ & GBS VII \\
\hline \multicolumn{7}{|c|}{ Fluorescence } \\
\hline Term & 0.19 & 0.61 & 0.55 & 0.45 & 0.58 & 0.74 \\
\hline \multicolumn{7}{|c|}{$\%$ of activated neutrophils } \\
\hline Term & -0.20 & 0.31 & 0.16 & 0.04 & 0.19 & 0.22 \\
\hline Preterm & -0.65 & -0.68 & -0.61 & -0.58 & -0.49 & -0.43 \\
\hline
\end{tabular}

S. epi, S. epidermidis; S. hae, S. haemolyticus; GBS, Group B streptococcus.

Stratification for term $(n=10)$ and preterm $(n=10)$ newborns. 
ide, and hydroxyl radicals (9). The intracellular production of superoxide and hydroxyl radicals in neutrophils of preterm newborns after stimulation with formylmethionyl-leucylphenylalanine or phorbol myristate acetate is reduced (14-17). Our study verifies that the oxidative burst intensity in preterm neutrophils is impaired also after stimulation with living bacteria and in a whole-blood system, including autologous humoral factors such as immunoglobulins and complement.

We found a tendency toward a lower percentage of oxidative active neutrophils after bacterial stimulation in preterm compared to term newborns, but this difference was not statistically significant. In view of the highly significant difference in oxidative burst intensity between the two groups of newborns, this might indicate that the main difference between term and preterm neutrophils lies in the efficacy of the intracellular bactericidal reactions after phagocytosis and not in the percentage number of phagocytosing active neutrophils. However, preterm newborns have significantly lower absolute numbers of PMNLs than term newborns (1). Under in vivo conditions, the number of phagocytosing active neutrophils is probably lower in preterm than in term newborns.

We found no difference between term and preterm newborns in the oxidative burst in unstimulated PMNLs. The correlation of the oxidative burst intensity between stimulated and unstimulated PMNLs observed in term newborns suggests regulated upgrading of the neutrophil activity after bacterial stimulation. In the preterm newborns, the oxidative burst intensity response seemed to be less organized and to show no correlation to the spontaneous activity. Furthermore, in preterm newborns, the correlation between the percentages of activated neutrophils before and after stimulation was negative. This might indicate an exhaustion phenomenon with a reduced ability of preterm newborns to maintain the numbers of phagocytosing active neutrophils over time.

Our study was not designed to compare adults and newborns, because one adult control participated in all experiments. The lower oxidative burst activity found in the adult control, however, is in accordance with earlier reports (21).

In the present study, differences were found between the oxidative burst responses to different bacterial strains, but the differences could not be correlated exclusively to the invasive capacity or slime-producing properties. The production of slime (biofilm) is reported to be a major virulence factor in $S$. epidermidis $(22,23)$, but most of the previous studies on this issue have addressed prosthetic infections in adult patients. Our results support the idea that the slime-producing capacity of $S$. epidermidis does not in itself result in escape from phagocytosis and an oxidative burst. This is in accordance with earlier reports on suspended CoNS $(2,6,24)$. The phenotypic variation in the production of slime according to environmental factors is well documented $(25,26)$, and the expression of slime on the nutrient-rich medium used in the oxidative burst assay might be depressed. However, the impact of slime as a virulence factor in neonatal CoNS sepsis is not clearly established, and other virulence factors may be of greater importance in preterm newborns.

Among the sepsis strains of $S$. epidermidis, one strain (94B080) belonged to the predominating genotype among the invasive CoNS isolates characterized in a former study (7) and thus possibly represented a more virulent clone. It is notable that this slime-negative strain of $S$. epidermidis evoked a lower oxidative burst than the sepsis strain of S. epidermidis, representing a unique and slime-positive isolate (94B105), and a response similar to that induced by GBS, a bacterial species that is highly virulent in neonates. One might speculate whether induction of a lower neutrophil oxidative response corresponds to a higher bacterial invasive capacity.

In a previous study, S. haemolyticus was found to infect the most preterm newborns (7). In the present study, that strain of S. haemolyticus (95B535) resulted in a comparably high oxidative burst in both term and preterm newborns. The selective capacity of $S$. haemolyticus to infect preterm newborns found in the previous study might be due to defects in other host defense mechanisms of importance at an earlier stage than the final intracellular bactericidal activity. Another example of the importance of earlier steps in the host defense might be the two contaminant strains of S. epidermidis: despite the induction of a low oxidative burst response (similar to that to bacteria with a high invasive capacity), they were clinically proved to be noninvasive.

The final neutrophil intracellular bacterial killing depends on the interaction between the oxidative burst and proteolytic and cationic proteins released from the neutrophil primary granulae into the phagosome (27). Reeves et al. (27) recently demonstrated that the oxidative metabolites enhance release and activity of granulae proteins by activation of $\mathrm{K}+$ influx into the phagosome and also suggest that the interaction between the oxidative and protein bactericidal systems might be of importance for a balanced neutrophil response, thus minimizing undesired tissue damage by uncontrolled release of neutrophil oxidative and proteolytic products. Our study demonstrates a reduced and perhaps less organized oxidative response in preterm PMNLs after bacterial challenge. The protein content in neonatal neutrophil granulae compared to adults differs in different proteins. For an example, preterm neutrophils show a reduced release of bactericidal/permeability-increasing protein, which is essential in the defense toward Gram-negative bacteria (28), whereas the release of myeloperoxidase is similar in newborns compared with adults $(14,15,28)$. A lower bactericidal capacity of CoNS in preterm PMNLs compared to term newborns or adults has been reported (2), but studies of the correlation between neonatal neutrophil oxidative burst and bactericidal capacity in response to CoNS remains to be carried out. The oxidative burst assay used in the present study proved to be suitable for neonatal studies. Further investigations of the interaction between the oxidative and proteolytic bactericidal mechanisms in neonatal neutrophils are of interest.

\section{REFERENCES}

1. Carr R 2000 Neutrophil production and function in newborn infants. Br J Haematol 110:18-28

2. Schutze GE, Hall MA, Baker CJ, Edwards MS 1991 Role of neutrophil receptors in opsonophagocytosis of coagulase-negative staphylococci. Infect Immun 59:25732578

3. Bektas S, Goetze B, Speer CP 1990 Decreased adherence, chemotaxis and phagocytic activities of neutrophils from preterm neonates. Acta Paediatr Scand 79:1031-1038

4. Fuijiwara T, Kobayashi T, Takaya J, Taniuchi S, Kobayashi Y 1997 Plasma effects on phagocytic activity and hydrogen peroxide production by polymorphonuclear leukocytes in neonates. Clin Immunol Immunopathol 85:67-72 
5. Sohn AH, Garett DO, Sinkowitz-Cochran RL, Grohskopf LA, Levine GL, Stover BH, Siegel J, Jarvis WR 2001 Prevalence of nosocomial infections in neonatal intensive care unit patients: results from the first national point-prevalence survey. J Pediatr 139:821-827

6. Kristinsson KG, Hastings JGM, Spencer RC 1988 The role of extracellular slime in opsonophagocytosis of Staphylococcus epidermidis. J Med Microbiol 27:207-212

7. Björkqvist M, Söderquist B, Törnqvist E, Sjöberg L, Fredlund H, Kühn I, ColqueNavarro P, Schollin J 2002 Phenotypic and genotypic characterisation of blood isolates of coagulase-negative staphylococci in the newborn. APMIS 110:332-339

8. Gantz T, Lehrer RI 1997 Antimicrobial peptides of leukocytes. Curr Opin Hemato $4: 43-58$

9. Hampton MB, Kettle AJ, Winterbourn CC 1998 Inside the neutrophil phagosome: oxidants, myeloperoxidase and bacterial killing. Blood 92:3007-3017

10. Richardson MP, Ayliffe MJ, Helbert M, Davies EG 1998 A simple flow cytometry assay using dihydrorhodamine for the measurement of the neutrophil respiratory burst in whole blood: comparison with the quantitative nitrobluetetrazolium test. J Immunol Methods 219:187-193

11. Perticarari S, Presani G, Banfi E 1994 A new flow cytometric assay for the evaluation of phagocytosis and the oxidative burst in whole blood. J Immunol Methods 170:117124

12. Perticarari S, Presani G, Mangiarotti MA, Banfi E 1991 Simultaneous flow cytometric method to measure phagocytosis and oxidative products by neutrophils. Cytometry 12:687-693

13. Rothe G, Valet G 1990 Flow cytometric analysis of respiratory burst activity in phagocytes with hydroethidine and 2'7'-Dichlorofluorescein. J Leukoc Biol 47:440448

14. Ambruso DR, Bentwood B, Henson PM, Johnston RB 1984 Oxidative metabolism of cord blood neutrophils: relationship to content and degranulation of cytoplasmic granules. Pediatr Res 18:1148-1153

15. Kjeldsen L, Sengeløv H, Lollike K, Borregaard N 1996 Granules and secretory vesicles in human neonatal neutrophils. Pediatr Res 40:120-129

16. Jaswon MS, Jones HM, Linch DC 1994 The effects of recombinant human granulocyte-macrophage colony stimulating factor on the neutrophil respiratory burst in the term and preterm infant when studied in whole blood. Pediatr Res 36:623-627
17. Drossou V, Kanakoudi F, Tzimouli V, Sarafidis K, Taparkou A, Bougiouklis D, Petrpoulou T, Kremenopoulus G 1997 Impact of prematurity, stress and sepsis on the neutrophil respiratory burst activity of neonates. Biol Neonate 72:201-209

18. Källman J, Schollin J, Håkansson S, Andersson A, Kihlström E 1993 Adherence of group B streptococci to human endothelial cells in vitro. APMIS 101:403-408

19. Mulder JG, Degener JE 1998 Slime-producing properties of coagulase-negative staphylococci isolated from blood cultures. Clin Microbiol Infect 4:689-694

20. Kragsbjerg P, Fogelqvist M, Fredlund H 2000 The effects of live Neisseria meningitidis and tumor necrosis factor- $\alpha$ on neutrophil oxidative burst and $\beta 2$-integrin expression. APMIS 108:276-282

21. Nishida A, Kimura H, Sugioka K, Nakano M 1990 The ability of granulocytes to generate superoxide anions and hypochlorite during phagocytosis: comparison of neonatal granulocytes with adult granulocytes. Biol Neonate 58:145-151

22. Vuong C, Otto M 2002 Staphylococcus epidermidis infections. Microbes Infect 4:481-489

23. Frebourg NB, Lefebrve S, Baert S, Lemeland FF 2000 PCR-based assay for discriminating between invasive and contaminating Staphylococcus epidermidis strains. $\mathrm{J}$ Clin Microbiol 38:877-880

24. Heinzelmann M, Herzig DO, Swain B, Mercer-Jones MA, Bergamini TM, Polk HC Jr 1997 Phagocytosis and oxidative-burst response of planctonic Staphylococcus epidermidis RP62A and its non-slime-producing variant in human neutrophils. Clin Diagn Lab Immunol 4:705-710

25. Gelosia A, Baldassarri L, Deighton M, Nguyen T 2001 Phenotypic and genotypic markers of staphylococcus epidermidis virulence. Clin Microbiol Infect 7:193-199

26. Deighton M, Borland R 1993 Regulation of slime production in Staphylococcus epidermidis by iron limitation. Infect Immun 61:4473-4479

27. Reeves EP, Lu H, Messina CGM, Bolsover S, Gabella G, Potma EO, Warley A, Roes J, Segal AW 2002 Killing activity of neutrophils is mediated through activation of proteases by $\mathrm{K}^{+}$influx. Nature 416:291-297

28. Nupponen I, Turunen R, Nevalainen T, Peuravuori H, Pohjavuori M, Repo H, Andersson S 2002 Extracellular release of bactericidal/permeability-increasing protein in newborn infants. Pediatr Res 51:670-674 